\title{
O trabalho das enfermeiras no Programa de Saúde da Família em Marília/SP
}

\author{
NURSES' ROLE IN THE FAMILY HEALTH PROGRAM IN MARÍLIA/SP \\ EL TRABAJO DE LAS ENFERMERAS EN EL PROGRAMA DE SALUD \\ DE LAFAMILIA EN MARÍLIA/SP
}

Regina Célia Ermel1', Lislaine Aparecida Fracolli²

\section{RESUMO}

A estratégia de Saúde da Família apresenta-se como uma alternativa de superação do paradigma dominante no campo da saúde. Este trabalho teve como objetivo identificar o processo de trabalho das enfermeiras no Programa Saúde da Família (PSF) no município de Marília, SP. A metodologia utilizada baseou-se nos pressupostos da Teoria de Intervenção Práxica em Enfermagem em Saúde Coletiva proposto por Egry (1996). Foram sujeitos do estudo a totalidade de enfermeiras que atuavam no PSF do município de Marília, no ano de 2001. Os dados foram coletados por meio de entrevista semi-estruturada e de observação direta do trabalho da enfermeira. Os resultados apontaram que as enfermeiras do PSF são jovens e possuem pouca experiência de trabalho em atenção básica. $\mathrm{O}$ processo de trabalho, desenvolvido por estas profissionais, toma como objeto o corpo individual, têm por finalidade os perfis de desgaste dos grupos sociais e utilizam meios e instrumentos tradicionais da saúde pública.

\section{DESCRITORES}

Programa Saúde da Família. Papel do profissional de enfermagem.

Enfermagem em saúde pública.

\section{ABSTRACT}

The Family Health strategy presents itself as an alternative for overcoming the dominant paradigm in the health field. This study aims at identifying the work process of nurses in the Family Health Program (FHP) in the city of Marília, in the State of São Paulo. The methodology used is based on the Theory of Praxic Intervention in Nursing in General Health proposed by Egry (1996). Every nurse who participated in the FHP in Marília in 2001 was considered subject of the study. The data was collected through semi-structured interviews and direct observation of the nurses' work. The results show that nurses in the FHP are young and have little experience in basic health care. The work process developed by these professionals has as its object the individual body, is aimed at showing the patterns of stress of the social groups and makes use of methods and ordinary instruments of public health.

\section{KEY WORDS}

Family Health Program.

Nurse's role.

Nursing in public health.

\section{RESUMEN}

La estrategia de Salud de la Familia se presenta como una alternativa de superación del paradigma dominante en el campo de la salud. Este trabajo tuvo como objetivo identificar el proceso de trabajo de las enfermeras en el Programa Salud de la Familia (PSF) en el municipio de Marília, SP. La metodología utilizada se basó en los presupuestos de la Teoría de Intervención Práxica en Enfermería en Salud Colectiva propuesta por Egry (1996). Los sujetos de estudio fueron la totalidad de enfermeras que actuaban en el PSF del municipio de Marília, en el 2001. Los datos fueron recolectados por medio de una entrevista semi-estructurada y de observación directa del trabajo de la enfermera. Los resultados señalaron que las enfermeras del PSF son jóvenes, poseen poca experiencia de trabajo en atención básica. El proceso de trabajo desarrollado por estas profesionales toma como objeto el cuerpo individual, tiene por finalidad los perfiles de desgaste de los grupos sociales y utilizan medios e instrumentos tradicionales de salud pública.

\section{DESCRIPTORES}

Programa Salud de la Familia.

Rol de la enfermera.

Enfermería en salud pública.
1 Enfermeira. Mestre em Enfermagem em Saúde Coletiva pela Escola de Enfermagem da Universidade de São Paulo (EEUSP). Professora junto ao curso de Enfermagem e Obstetrícia da Universidade de Marília - UNIMAR, SP. rcermel@terra.com.br 2 Enfermeira. Professora Doutora do Departamento de Enfermagem em Saúde Coletiva da EEUSP. lislaine@usp.br. 


\section{INTRODUÇÃO}

O Ministério de Saúde brasileiro na tentativa de operacionalizar um modelo tecno-assistencial, pautado nos princípios do Sistema Único de Saúde (SUS) propõe, em 1994, o Programa de Saúde da Família (PSF) como uma estratégia para a reorganização da produção de cuidados de saúde, que tem como objetivo reorientar a prática assistencial em direção a uma assistência à saúde centrada na família, entendida e percebida a partir de seu ambiente físico e social.

O PSF elege como diretrizes operacionais os princípios: caráter substitutivo de suas práticas (substituição das práticas convencionais de assistência por um novo processo de trabalho, centrado na Vigilância à Saúde); integralidade e hierarquização das ações (a unidade de saúde da família está inserida no primeiro nível de ações e serviços do sistema local de saúde); territorialização e adscrição de clientela (o trabalho das equipes é desenvolvido em um território definido); e trabalho em equipe multiprofissional (a unidade produtiva do PSF é a Equipe de Saúde da Família, composta minimamente por um médico generalista, um enfermeiro, um auxiliar de enfermagem e de quatro a seis agentes comunitários de saúde) ${ }^{(1)}$.

A estratégia do PSF está estruturada na lógica de atenção básica à saúde, gerando novas práticas setoriais e afirmando a indissociabilidade entre os trabalhos clínicos e a promoção da saúde. Para tanto, necessita desenvolver processos de trabalho que estabeleçam uma nova relação entre os profissionais de saúde e a comunidade, estes processos de trabalho devem se traduzir, especialmente, em termos de desenvolvimento de ações humani-zadas, tecnicamente competentes, intersetorialmente articuladas, e socialmente apropriadas $^{(2)}$.

No Estado de São Paulo, a inserção da enfermeira nos serviços de atenção básica tem início a partir da década de 70, com a Reforma Administrativa da Saúde, que institui o modelo da Programação em Saúde. Esse modelo tecnológico caracterizava-se pela implantação e diversificação da assistência médica individual, prestada pela secretaria estadual de saúde. A assistência à saúde, até então polarizada entre médicos e atendentes ou educador sanitário ou visitador passou a ser intermediada pela presença da enfermeira. Couberam a essas profissionais as atividades de supervisão, treinamento, controle e coordenação do pessoal de enfermagem, tendo sido designadas, também, para atuar na vigilância epidemiológica, nas reuniões com grupos da comunidade, na supervisão da visita domiciliária e nas atividades educativas. Na realidade, a enfermeira foi incorporada na atenção básica para desenvolver atividades predominantemente gerenciais, o que acarretou conflitos para essas profissionais ${ }^{(3)}$.

A partir de meados da década de 80 , com a centralidade do pronto atendimento como modalidade de organização da atenção básica, o trabalho da enfermeira em Saúde Pública passou a se caracterizar, principalmente, como auxílio ao pronto atendimento médico, pautado num recorte clínico e individual, tornando-se rara a realização de ações de caráter coletivo $^{(4)}$.

Com a implantação do SUS, no final da década de 80 , os serviços públicos de saúde passam por um processo de revisão do modelo assistencial de maneira que, novas práticas se instituem e outras são abandonadas. Em meados da década de 90, a estratégia do PSF recoloca o debate da necessidade de mudança do modelo assistencial, na perspectiva de construção de um modelo que tome como objeto o processo saúde/doença em sua dimensão coletiva, e, consequentemente, busque construir práticas assistenciais estruturadas em novos paradigmas $^{(5)}$. No PSF, a prática de enfermagem se insere buscando a reformulação e a integração de ações com os demais trabalhadores da equipe de saúde. Esta reformulação e integração acontecem, principalmente, através de um processo de articulação das intervenções técnicas e da interação entre os outros profissionais da Equipe de Saúde da Família (ESF). Assim, para a compreensão da especificidade do trabalho da enfermeira no PSF é imprescindível que se analise a sua inserção no trabalho da equipe, o modo como se dá sua relação com o processo de trabalho e o modo como a enfermeira realiza o cuidado específico de enfermagem ${ }^{(6)}$.

O trabalho da enfermeira no PSF se constitui: no monitoramento das condiç̃es de saúde, como núcleo da atenção de enfermagem, seja no atendimento individual ou no atendimento grupal; no levantamento e monitoramento de problemas de saúde (seja no enfoque de risco ou de vulnerabilidade) sendo que estes, deverão estar articulados à intervenção nos agravos de ordem patológica (e portanto pautados no saber da clínica) e no exercício de uma prática de enfermagem comunicativa, no sentido dialógico e emancipatório, buscando a ampliação da autonomia dos sujeitos ${ }^{(6)}$. Assim, falar das práticas de enfermagem pressupõe o entendimento de que a enfermagem, enquanto prática social, busca sempre responder às exigências sociais e de saúde de uma determinada época e de um determinado espaço social. Frente a isso o objetivo deste estudo é caracterizar o processo de trabalho da enfermeira no PSF, identificando o objeto, a finalidade, os meios e instrumentos do mesmo. 


\section{MÉTODO}

\section{Quadro teórico}

As bases teóricas para a realização desta pesquisa são as concepções de Determinação Social do Processo Saúde Doença e Processo de Trabalho. O processo saúde doença, neste estudo, é entendido como um processo histórico e dinâmico, isto é, determinado pela forma como cada indivíduo se insere no modo de produção dominante na estrutura social a que pertence. É a síntese da totalidade das determinações que operam sobre a qualidade de vida social, e está articulado ao aspecto econômico, político, social, de relacionamento familiar e de responsabilidade humana. O processo saúde-doença aproxima o pólo doença, não apenas dos sofrimentos físicos, mas também, dos psíquicos, morais e éticos. Além disso, tem relação com a capacidade vital, perfil de morbidade e mortalidade, articulando-se ao processo de desenvolvimento e crescimento do indivíduo. $\mathrm{O}$ processo saúde-doença, portanto, não é um processo individual, tampouco se refere exclusivamente à dimensão biológica do homem. É um processo particular de uma sociedade que expressa, ao nível individual, as condições coletivas de vida, resultantes das características concretas dos perfis de produção e dos perfis de consumo e as consequiências destes perfis nas diferentes formas de vida ${ }^{(7)}$.

A concepção de trabalho utilizada neste estudo considera que o trabalho é uma atividade humana, realizada por um grupo de pessoas que a ela se dedica e, assim, reproduz sua existência humana. $\mathrm{O}$ trabalho é determinado por uma necessidade ou carência, é o meio de subsistência para a satisfação das necessidades materiais e não materiais, e também possibilita a livre criação. Assinala-se que dadas as características do trabalho humano (produzir sempre considerando as necessidades sociais, relacionar os homens através de seus produtos, relacioná-los conforme o grau de domínio que eles têm - ou deixam de ter - das condições de trabalho), este é, antes de tudo, o processo de produção e reprodução do homem social, historicamente determinado através da produção de bens e serviços ${ }^{(8)}$. O processo de trabalho é constituído por três elementos básicos: objeto, os meios ou instrumentos e a finalidade. O objeto é a matéria em que o trabalho se aplica, os meios ou instrumentos são coisas que se inserem entre o trabalhador e o objeto. A finalidade refere-se à atividade orientada a um fim ou o que se deseja obter. Referido de outra forma,

um objeto de trabalho é um fragmento da realidade recortado por um olhar que concebe, intelectualmente, um produto. A atividade do homem opera uma transformação, subordinada a um determinado fim no objeto sobre o qual atua por meio do instrumental de trabalho. Os instrumentos ou meios de trabalho permitem a aproximação e transformação do objeto. As características do objeto, de um lado, e a finalidade do trabalho, de outro, determinam as características dos meios de trabalho(8).
Buscando apreender o trabalho da enfermeira no PSF e contribuir para sua transformação, o presente estudo utilizou a Teoria de Intervenção Práxica de Enfermagem em Saúde Coletiva (TIPESC) ${ }^{(7)}$. A compreensão acerca dos determinantes da saúde das populações, assim como a discussão e a formulação de políticas de saúde têm sido grandemente prejudicadas pela perpetuação de instrumentos de interpretação incompletos, obsoletos ou equivocados ${ }^{(9)}$. A TIPESC propõe uma forma sistematizada para captar, interpretar e intervir no processo saúde-doença, tendo em vista suas manifestações nas dimensões singular, particular e estrutural da realidade. A dimensão singular, relativa aos indivíduos e suas famílias, é aquela onde em última instância manifestam-se os processos que levam ao adoecimento e à morte, como expressão das formas de reprodução social de grupos sociais distintos, ou seja, a dimensão particular. Ambos articulam-se aos processos de produção e reprodução social da coletividade em que se inserem, cujo contexto e estrutura social são historicamente determinados, constituindo assim a dimensão estrutural. À captação, interpretação e intervenção seguir-se-ia a contínua re-interpretação do fenômeno (processo saúde-doença), de forma práxica, constituindo a unidade dialética teoria-prática ${ }^{(9)}$.

A TIPESC, na sua vertente metodológica, propõe uma sistematização para se captar e interpretar o processo saúdedoença, de forma dinâmica e articulada aos processos de produção e reprodução social, o que possibilita que as questões relativas a esse processo em uma dada coletividade sejam relacionadas ao contexto social e histórico que as determinou. É composta dos seguintes passos: captação da realidade objetiva, interpretação da realidade objetiva, intervenção na realidade e re-interpretação da realidade.

Para essa pesquisa utilizaram-se apenas os dois primeiros passos da TIPESC uma vez que não era objetivo dessa pesquisa intervir diretamente na realidade do município de Marília. Utilizaram-se os instrumentos de captação da realidade e análise da realidade proposta pela TIPESC, os quais buscam o estabelecimento dos nexos explicativos entre as diferentes dimensões que compõe a realidade (singular, particular e estrutural).

\section{O cenário de estudo}

O campo empírico para desenvolvimento dessa pesquisa foi o PSF do Município de Marília/SP, no ano de 2001. O Município de Marília localiza-se na região Centro-Oeste do Estado de São Paulo e conta com cerca de 190.000 habitantes. O processo de municipalização da saúde nesse município iniciou-se em 1988, e dez anos após, o Município de Marília assumiu a gestão plena do sistema de saúde municipal. Assim, em 1998, o município era responsável pelo processo de gestão de todo o sistema municipal de saúde, compreendendo além da rede básica de saúde e das ações de saúde coletiva, todo o processo de organização e regulação 
dos demais níveis de atenção à saúde. O PSF foi implantado nesse município em 1998, desde então à Secretaria Municipal de Higiene e Saúde (SMHS) vem empreendendo esforços na consolidação desse modelo. A estratégia adotada, pelo gestor municipal, é de complementar o modelo municipal de saúde vigente com o PSF, priorizando a atenção fundamentada na extensão dos cuidados básicos às populações mais carentes, com maior dificuldade de acesso e que apresentem maiores riscos em relação à sua situação de saúde. No município de Marília, as Unidades de Saúde da Família (USF) deveriam atuar de forma integrada à rede básica de saúde contando com o apoio das Unidades Básicas de Saúde (UBS) da região de abrangência ${ }^{(10)}$. A estratégia do PSF, nessa localidade, prioriza as ações de prevenção, promoção e recuperação da saúde das pessoas de forma integral e contínua $^{(11)}$.

Em 2001, Marília contava com oito (8) equipes de Saúde da Família, sendo seis (6) localizadas em área urbana e duas (2) em área rural. As equipes estavam sob a supervisão técnica e administrativa da SMHS. Cada equipe responsabilizava-se pela Vigilância à Saúde de 600 a 1000 famílias. Nesse período, a cobertura da população com o PSF era $11 \%$, os profissionais das equipes de família residiam no município e trabalhavam em regime de dedicação exclusiva ${ }^{(11)}$. As Equipes de Saúde da Família (ESF) eram compostas por um médico, uma enfermeira, um dentista, dois auxiliares de enfermagem, seis agentes comunitários de saúde, um auxiliar de serviços gerais, um auxiliar de consultório dentário e um motorista. As atividades desenvolvidas pelas equipes eram: cadastrar as famílias da área de atuação; conhecer a realidade das famílias, identificando seus principais problemas e situações de risco; elaborar o plano de saúde local; organizar as ações de saúde para o atendimento individual e coletivo; prestar assistência integral na USF, na comunidade e no domicílio; desenvolver ações educativas e intersetoriais no enfrentamento dos problemas da comunidade; participar de programa de educação continuada em serviço para melhorar a qualidade do trabalho ${ }^{(11)}$.

Ao definir o trabalho da enfermeira no PSF, a SMHS de Marília compila as propostas ministeriais, e propõe que estas desenvolvam seus processos de trabalho em dois campos essenciais:

na USF, junto à equipe de profissionais; e na comunidade, apoiando e supervisionando o trabalho dos Agentes Comunitários de Saúde (ACS), bem como assistindo as pessoas que necessitam de atenção de enfermagem ${ }^{(2)}$.

Considera que as enfermeiras devam ainda executar,

no nível de suas competências, ações de assistência básica, de vigilância epidemiológica e sanitária nas áreas de atenção à criança, ao adolescente, à mulher, ao trabalhador e ao idoso; desenvolver ações para a capacitação dos ACS e auxiliares de enfermagem; oportunizar os con- tatos com indivíduos sadios ou doentes, visando promover a saúde e abordar os aspectos de educação sanitária; promover a qualidade de vida e contribuir para que o meio ambiente torne-se mais saudável; discutir, junto à equipe de trabalho e comunidade, o conceito de cidadania, enfatizando os direitos de saúde e as bases legais que os legitimam; participar do processo de programação e planejamento das ações e da organização do processo de trabalho nas USF(11).

Assim, o PSF para ser implementado necessita que as enfermeiras incorporem às suas práticas ações de assistência e promoção da saúde e desenvolvam um processo de trabalho específico dentro das equipes de saúde da família.

\section{Sujeitos do estudo e instrumentos utilizados}

Foram sujeitos desse estudo oito (8) enfermeiras que trabalhavam no PSF, no município de Marília e o gestor municipal do PSF, no ano de 2001. Os dados foram coletados através da observação direta do trabalho das enfermeiras e através de entrevistas semi estruturadas, com o gestor municipal e com as enfermeiras. O presente estudo foi encaminhado ao Comitê de Ética em Pesquisa Envolvendo Seres Humanos da Escola de Enfermagem da USP de São Paulo e foi aprovado.

\section{Descrição dos procedimentos desenvolvidos para a coleta de dados}

Os dados foram coletados nos meses de outubro, novembro e dezembro de 2001, nas USF do Município de Marília/ SP. Foram realizadas seis (6) observações de enfermeiras, em atuação no campo, a enfermeira era contatada individualmente pelo pesquisador o qual solicitava a esta que agendasse um dia para recebê-lo na USF, para que este pudesse observá-la durante um período do seu dia de trabalho (normalmente pela manhã). Cada observação durava cerca de quatro horas e algumas vezes, era possível observar-se duas atividades da enfermeira (por exemplo Consulta de Enfermagem e Visita Domiciliária, ou Grupo Educativo e Consulta Enfermagem, etc.); outras vezes apenas uma atividade, a depender da demanda para a enfermeira naquele dia. Duas (2) enfermeiras não puderam ter suas atividades observadas, pois todas as vezes que agendaram com o pesquisador aconteceu um imprevisto que as obrigava a sair da USF. As entrevistas foram realizadas com a totalidade das enfermeiras e com o gestor, em um ambiente privado, e agendadas com antecedência. As entrevistas foram gravadas, as fitas transcritas e seu conteúdo utilizado para análise. Para manter o anonimato dos entrevistados, optou-se por identificar as respostas com códigos (E1, referindo-se à primeira enfermeira entrevistada, até E8; e G para o gestor). Solicitou-se aos sujeitos que assinassem o termo de consentimento para participação na pesquisa. 


\section{Organização e Análise dos dados}

Precedeu a realização das entrevistas com as enfermeiras, o preenchimento de um formulário pelas mesmas, o que permitiu traçar um perfil do grupo que integrou este estudo. Os registros das observações foram compilados em um diário de campo. Os textos originados das fitas transcritas foram ordenados e fichados e após leitura exaustiva seus conteúdos eram assinalados. Os dados foram analisados seguindo a articulação entre as dimensões (singular, particular e estrutural) proposta pela TIPESC e a técnica de Análise de Conteúdo ${ }^{(12)}$.

\section{RESULTADOS E DISCUSSÕES}

\section{A organização técnica e política do PSF no município de Marília: a perspectiva do gestor municipal}

Em relação a atenção básica, o município tem o modelo de pronto atendimento que, segundo o gestor, é um modelo que vem sendo questionado por ser pouco resolutivo. Com a finalidade de contribuir para a resolubilidade do modelo, incorporou-se o PSF no município procurando, com essa estratégia, melhorar a qualidade da relação profissional de saúde/usuário do serviço. A coordenação do PSF, no município, fica a cargo de um gestor que estabelece diretrizes, define as necessidades de capacitação e de apoio técnico sendo, o gestor, o elo de ligação entre as equipes e as demais secretarias da Prefeitura de Marília. Para o gestor do PSF, o impacto do programa na qualidade da assistência à saúde prestada à população ainda não pôde ser inteiramente avaliado, contudo tem-se verificado a alteração de alguns indicadores de saúde nas áreas onde o programa está implantado. Na opinião do gestor do PSF, o trabalho da enfermeira nesse programa deve se constituir de ações como consulta e prescrição de enfermagem, supervisão dos ACS, participação em reuniões de equipe e questões administrativas. Para o gestor, a enfermeira deve ter um perfil de liderança, ser criativa, estar envolvida com a realidade social, ser comunicativa, ter disponibilidade, estrutura emocional boa, não precisando ser especialista em Saúde Coletiva.

\footnotetext{
.. a enfermeira deve fazer consulta de enfermagem, consultar o paciente, fazer uma prescrição de enfermagem, a gente precisou fazer uma capacitação pelo pólo de capacitação da Faculdade de Medicina, a gente fez uma capacitação de estado de saúde do indivíduo e a gente resgatou este papel... (G).
}

...ela deve ainda responder pelo material, pela limpeza da unidade, a questão administrativa da unidade fica sob sua responsabilidade... (G).

\section{O perfil profissional das enfermeiras que atuam no PSF em Marília/SP}

As enfermeiras do PSF de Marília/SP situam-se na faixa etária compreendida entre 24 e 31 anos. Possuem de 3 a 7 anos de formadas em enfermagem e a maioria (seis) trabalha no PSF no mínimo há 1 ano e no máximo há 3 anos. Com relação à qualificação profissional, três enfermeiras não possuíam nenhum tipo de especialização, duas possuíam especialização na área de Saúde Mental, uma era especialista em Administração Hospitalar e uma em Administração de Serviços de Saúde. Chama a atenção o fato de que nenhuma enfermeira contratada para trabalhar no PSF possuía especialização na área de Saúde Coletiva ou Saúde Pública. A maioria das enfermeiras foi recrutada no próprio município, são contratadas em regime de CLT, com carga horária semanal de 40 horas e salário de $\mathrm{R} \$ 1.300,00$, estando vinculadas à instituições filantrópicas como o Hospital São Francisco de Assis e a Maternidade Gota de Leite.

\section{O trabalho da enfermeira no PSF: a identificação do objeto de intervenção}

O objeto de trabalho das enfermeiras foi identificado a partir das observações no cotidiano de seus serviços. As enfermeiras foram observadas realizando ações de visita domiciliária (VD) e consulta de enfermagem (CE). Com relação à VD, pode-se observar que o objeto de intervenção, recortado pela enfermeira, é o indivíduo doente e embora as enfermeiras afirmem que a VD é para a família, o enfoque da mesma é no indivíduo. A família é abordada como alguém que vai contribuir para a terapêutica. A enfermeira, durante a VD, não busca identificar a dinâmica familiar e tampouco discute as mudanças na vida da família, face ao problema de saúde de um de seus membros. Na VD os aspectos sociais e financeiros são vistos como fatores que impedem a terapêutica. Com relação à $\mathrm{CE}$, a enfermeira toma como objeto de sua intervenção o indivíduo, não traz para dentro da CE a dimensão social e a postura que adota é prescritiva. Não é uma postura dialógica de reconhecimento, de intervenção sobre o problema. Encara a CE como uma forma de aliviar a agenda do médico e não aborda a família. $\mathrm{Na} \mathrm{CE}$, as enfermeiras costumam transferir para o indivíduo a culpa por não executar o tratamento adequadamente, sendo que o social e o econômico não são discutidos enquanto determinantes do processo saúde-doença dessa família.

\footnotetext{
...a gente tem o costume de estar agendando estas visitas. É uma família que precisa de acompanhamento devido às patologias - diabetes, hipertensão - de seus membros. Eu avalio não só a nível da patologia, o nível social também (E2).

\begin{abstract}
...e para o trabalho da equipe é muito melhor ter $C E$, porque se fosse agendar tudo isso para o médico, as vezes é um caso simples, que acaba acumulando a agenda do médico e não tem necessidade se tem enfermeira (E6).
\end{abstract}

\section{O trabalho da enfermeira no PSF: a identificação dos instrumentos e da finalidade de intervenção}

As enfermeiras que atuavam no PSF de Marília, ao serem questionadas sobre o conhecimento que consideravam ser 
necessário para sua prática, responderam que precisavam apreender: tecnologias sistematizadas para intervenção de enfermagem (sistematização da assistência de enfermagem); técnicas relativas à realização do exame físico; consulta de enfermagem para a assistência a patologias crônicas e transmissíveis; vacinação; assistência de enfermagem a emergências e técnicas específicas de enfermagem (curativo; administração de medicamentos; entre outros). As ações desenvolvidas pelas enfermeiras estavam concentradas no processo de trabalho de assistência, dirigiam-se para os indivíduos ou grupo de indivíduos com determinada patologia; envolviam, principalmente, a realização de: consulta de enfermagem para a mulher (voltada para a coleta de citologia oncológica, acompanhamento pré-natal e puerpério) e para a criança (com enfoque na puericultura); atendimento a grupos de puericultura, hipertensão, diabetes; visita domiciliária para indivíduos acamados; acolhimento na unidade de saúde. As enfermeiras executavam, também, algumas ações de gerência, tais como supervisão e treinamento de auxiliares de enfermagem e ACS; previsão e provisão de material; elaboração de relatórios e boletins mensais para o Sistema de Informação em Atenção Básica (SIAB).

De um modo geral, as enfermeiras investigadas consideravam que sua intervenção no PSF deveria estar centrada nos perfis de desgaste dos grupos sociais. Possuíam uma visão reduzida sobre os problemas de saúde de sua região e necessitavam ampliar suas concepções sobre perfil epidemiológico, incorporando o conhecimento dos perfis de produção e reprodução social da sua comunidade ${ }^{(12)}$. Quando instigadas a dar sugestões sobre o trabalho da enfermeira no PSF, responderam que a enfermeira precisa: estar envolvida com a comunidade, saber trabalhar em grupo, ser preparada, ainda na graduação, para trabalhar em equipe e trabalhar com grupos, se moldar às características do local e do grupo de trabalho, gostar do que faz e ampliar o sentido de classe profissional.

Principalmente exame físico de uma maneira geral, é preciso ter muito conhecimento do exame físico, dos processos de enfermagem para a gente estar aplicando (E1).

... e este conhecimento é técnico mesmo, do que é uma verminose, o que é o resultado do papanicolaou, estar por dentro das patologias para a gente poder dar uma assistência melhor.... (E6).

... as necessidades curativas a gente presta, mas precisam mais da questão educativa. Mas suprir todas as necessidades da comunidade a gente não tem como (E1).

\section{O trabalho em equipe no PSF : a visão da enfermeira}

Com relação ao trabalho em equipe no PSF, 62,5\% das enfermeiras entrevistadas relataram encontrar dificuldades de interação com a equipe e consideram que estas dificuldades são de ordem pessoal (quando trabalham com pessoas que pensam de maneira muito diferente); de ordem profissional (porque cada profissional tem sua característica própria ou advém de diferentes estratos sociais). Muitas vezes, ao se trabalhar em equipe, pode ocorrer sobreposição de função com outro profissional de saúde. Quando inquiridas sobre esse fato, $50 \%$ das enfermeiras relataram que realizam ações de outros profissionais de saúde, citando, principalmente, o psicólogo, o assistente social, o auxiliar de enfermagem e o ACS. Tal constatação aponta a necessidade de revisão da equipe mínima do PSF ou a criação de equipes multiprofissionais de referência.

\begin{abstract}
Uma particularidade da nossa equipe é que foi uma luta para eu montar uma equipe, não foi fácil, conviver com 10 , 12 pessoas é difícil, foi preciso fazer demissões até encontrar cada membro com perfil adequado... A gente tem casos de pessoas com péssimo humor, não conseguem se relacionar com os colegas. No trabalho em equipe cada um tem que ajudar o outro, precisa aceitar que erra, pedir desculpa, tentar corrigir o erro, a grande dificuldade de montar esta equipe é conseguir as pessoas que têm este perfil...(E4).
\end{abstract}

\section{CONSIDERAÇÕES FINAIS}

A finalidade dessa pesquisa foi compreender o trabalho que a enfermeira vem desenvolvendo nas Equipes de Saúde da Família no sentido de contribuir para a transformação dessas práticas, tendo por referência o Sistema Único de Saúde e o Programa Saúde da Família.

As averiguações explicitaram que no Programa Saúde da Família, em Marília, as enfermeiras tomam como objeto de seu trabalho o corpo individual, como finalidade a intervenção nos perfis de desgaste dos grupos sociais e operam nesse processo de trabalho com instrumentos tradicionais da saúde pública, tais como a Consulta de Enfermagem e a Visita Domiciliária. A análise dos dados mostrou ainda que as enfermeiras do PSF em Marília/SP, ao exercitarem sua prática, reiteram a lógica da prática clínica, individual, curativa e atuam tomando por referência a teoria da multicausalidade do processo saúde-doença.

O PSF em Marília, ao propor um novo modo de operar o trabalho em saúde, caminha para um processo de trabalho centrado na Vigilância à Saúde. A enfermeira, para atuar na lógica da Vigilância à Saúde, precisa executar ações de monitoramento dos problemas e necessidades de saúde dos diferentes grupos sociais, e deve considerar como objeto de sua ação os perfis de saúde-doença desses grupos e as condições que desencadeiam os processos de adoecimento/ fortalecimento dos mesmos. Para operar na lógica da Vigilância a Saúde, as enfermeiras no PSF de Marília, precisariam buscar implementar uma articulação entre a clínica e a epidemiologia na prestação de cuidados aos indivíduos/ coletivos $^{(12)}$. 
Um outro aspecto do trabalho da enfermeira, que merece ser discutido, refere-se à finalidade do trabalho dessas profissionais no PSF. O trabalho da enfermeira no PSF tem como finalidade o cuidado de enfermagem e a criação e manutenção de condições adequadas para a execução do cuidado ${ }^{(6)}$. Assim, a intervenção nos perfis de desgaste dos indivíduos/grupos precisa ser revisto enquanto finalidade do trabalho das enfermeiras nas equipes de PSF em Marília. A atuação de enfermagem, que tem por finalidade o cuidado, não se reduz à espera de sinais de alarme ou dos denominados eventos sentinela, mas se concretiza na detecção precoce de estrangulamentos ou de nós críticos que evidenciam os problemas e as necessidades de saúde das pessoas sob sua responsabilidade $^{(11)}$. Este estudo nos permitiu concluir que há necessidade de se desenvolver processos de educação em serviço, para as enfermeiras que atuam no PSF em Marília, que possibilitem a apreensão de instrumentos adequados para intervir nos problemas/necessidades de saúde dos indivíduos/coletivos a partir do conceito da determinação social do processo saúde-doença.

\section{REFERÊNCIAS}

(1) Brasil. Ministério da Saúde. Saúde da família: uma estratégia para a reorientação do modelo assistencial. Brasília; 1997.

(2) Brasil. Ministério da Saúde. Secretaria de Políticas de Saúde. A implantação da unidade de saúde da família. Brasília; 2000.

(3) Villa TCS, Mishima SM, Rocha SMM. A enfermagem nos serviços de saúde pública do Estado de São Paulo. In: Almeida MCP, Rocha SMM, organizadores. O trabalho de enfermagem. São Paulo: Cortez; 1997. p. 27-60.

(4) Almeida MCP, Mishima SM, Silva EM, Mello DF. O trabalho de enfermagem e sua articulação com processo de trabalho em saúde coletiva: rede básica de saúde. In: Almeida MCP, Rocha SMM, organizadores. O trabalho de enfermagem. São Paulo: Cortez; 1997. p. 61-112.

(5) Mello C, Araújo MJS. A nova prática de enfermagem no Sistema Único de Saúde. In: Associação Brasileira de Enfermagem (ABEn). Descentralização em saúde e a prática de enfermagem: documento III. Brasília; 1992.

(6) Peduzzi M. A inserção do enfermeiro na equipe de saúde da família na perspectiva de promoção de saúde. In: Anais do $1^{\circ}$ Seminário Estadual: o enfermeiro no programa de saúde da família; 2000 nov. 9-11; São Paulo. São Paulo: Secretaria de Estado da Saúde; 2000. p. 1-11.
(7) Egry EY. Saúde coletiva: construindo um novo método de enfermagem. São Paulo: Icone; 1996.

(8) Gonçalves RBM. Tecnologias e organização social das práticas de saúde: características tecnológicas do processo de trabalho na rede estadual de centros de saúde de São Paulo: Hucitec; 1994.

(9) Oliveira MAC, Egry EY. A historicidade das teorias interpretativas do processo saúde-doença. Rev Esc Enferm USP. 2000;34(1):9-15.

(10) Marília. Secretaria Municipal de Higiene e Saúde. Diretrizes e propostas da secretaria municipal de higiene e saúde de Marília(SP) [online]. Marília; 2001. Disponível em: http://www.consaude. com.br/marilia/textos/III_Propostas_Sec.htm. [Acesso em 26 set. 2001].

(11) Oliveira MS. Modelos de atenção à saúde: PSF/PACS. Marília: Faculdade de Medicina de Marília; 2001.

(12) Bardin L. Análise de conteúdo. Lisboa: Edições 70; 1979.

(13) Fracolli LA, Bertolozzi MR. O perfil epidemiológico na prática do enfermeiro no Programa Saúde da Família. In: Brasil. Ministério da Saúde. Instituto para o Desenvolvimento da Saúde. Universidade de São Paulo. Manual de enfermagem. Brasília; 2001. p. 9-13. 